\title{
Supplemental Online Materials (SOM)
}

Here we present another form of Figure 11, in which the dust/IN effects (red lines) are presented based on the LoCCN\&NoDust case, meaning the results are for adding the dust layer to a non-dust condition. Therefore, the sign for the dust/IN effects in Figure S1 is opposite to that in Figure 11.

(a)

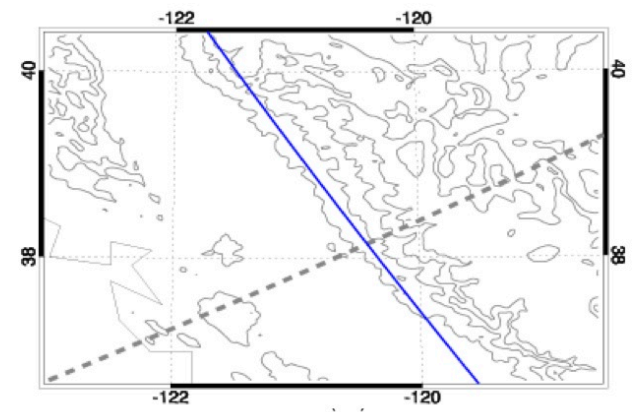

(b)

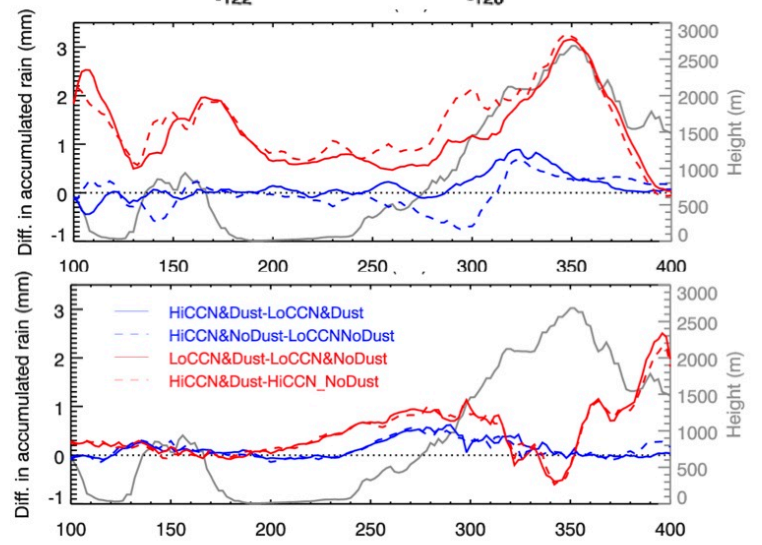

(d)

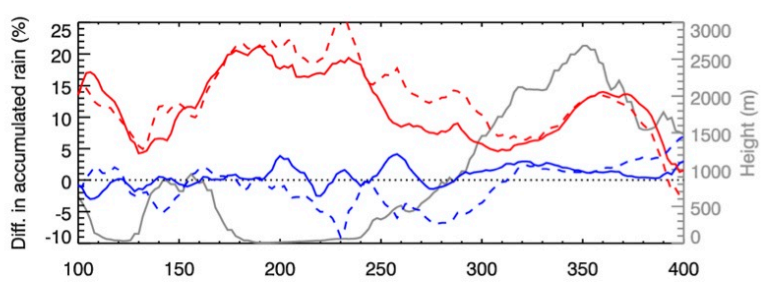

(e)

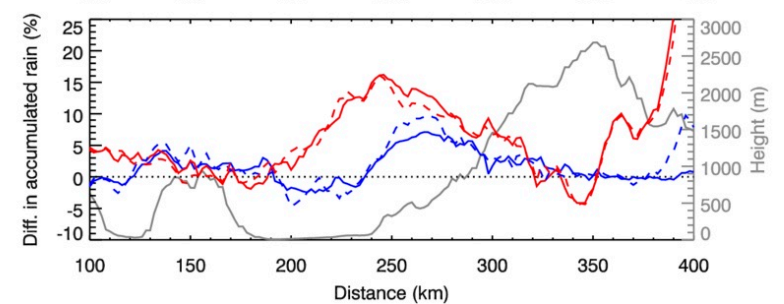

Fig. S1 Same as Figure 11, except the red lines denote dust effects by adding the dust/bio layer under the low CCN (solid; LoCCN\&Dust-LoCCN\&NoDust) and high CCN (dashed; HiCCN\&Dust-HiCCN\&NoDust) conditions. 